\title{
Circulating microRNA Profiles as Liquid Biopsies for the Characterization and Diagnosis of Fibromyalgia Syndrome
}

\author{
Andrea Masotti ${ }^{1}$ \&Antonella Baldassarre ${ }^{1}$ \&Maria Paola Guzzo ${ }^{2}$ \&Cristina Iannuccelli ${ }^{2}$ \& \\ Christian Barbato ${ }^{3} \&$ Manuela Di Franco ${ }^{2}$
}

Received: 27 August 2016 / Accepted: 17 October 2016

\# Springer Science+Business Media New York 2016

\begin{abstract}
This work was aimed at investigating the circulating microRNA (miRNA) profiles in serum and saliva of patients affected by fibromyalgia syndrome (FM), correlating their expression values with clinical and clinimetric parameters and to suggest a mathematical model for the diagnosis of FM. A number of 14 FM patients and sex- and age-matched controls were enrolled in our study. The expression of a panel of 179 miRNAs was evaluated by qPCR. Statistical analyses were performed in order to obtain a mathematical linear model, which could be employed as a supporting tool in the diagnosis of FM. Bioinformatics analysis on miRNA targets were performed to obtain the relevant biological processes related to FM syndrome and to characterize in details the disease. Six miRNAs were found downregulated in FM patients compared to controls. Five of these miRNAs have been included in a linear predictive model that reached a very high sensitivity $(100 \%)$ and a high specificity $(83.3 \%)$. Moreover, miR-
\end{abstract}

Andrea Masotti and Antonella Baldassarre equally contributed to the work.

Electronic supplementary material The online version of this article (doi:10.1007/s12035-016-0235-2) contains supplementary material, which is available to authorized users.

* Christian Barbato christian.barbato@cnr.it

* Manuela Di Franco manuela.difranco@uniroma1.it

1 Ospedale Pediatrico Bambino Gesù, Gene Expression-Microarrays Laboratory, V.le di San Paolo 15, 00146 Rome, Italy

2 Rheumatology Unit, Department of Internal Medicine and Medical Specialities, Sapienza University of Rome, Rome, Italy

3 Institute of Cell Biology and Neurobiology (IBCN), National Research Council (CNR), Via del Fosso di Fiorano, 64 00143 Rome, Italy
$320 \mathrm{~b}$ displayed a significant negative correlation $(r=-0.608$ and $p=0.036$ ) with ZSDS score. Finally, several biological processes related to brain function/development and muscular functions were found potentially implicated in FM syndrome. Our study suggests that the study of circulating miRNA profiles coupled to statistical and bioinformatics analyses is a useful tool to better characterize the FM syndrome and to propose a preliminary model for its diagnosis.

Keywords Fibromyalgia syndrome $\cdot$ Pain $\cdot$ Depression Circulating miRNAs $\cdot$ Liquid biopsies $\cdot$ Biomarkers

\section{Introduction}

Fibromyalgia (FM) is a syndrome characterized by chronic widespread pain, which affects about $2 \%$ of the general population, especially females. However, recent evidence has provided insights into the presence and impact of FM in children and adolescents [1,2] and widespread pain has a prevalence from 2 to $6 \%$ in schoolchildren [3]. In 1990, the criteria of the American College of Rheumatology (ACR) defined FM by the presence of widespread pain for more than 3 months and tenderness on palpation of at least 11 of 18 painful points called tender points (TPs) [4]. The new ACR criteria (2010) did not recommend to consider TPs for the diagnosis, but other two parameters: the widespread pain index and the symptom severity scale score which considers somatic and cognitive symptoms [5].

Despite numerous studies, the pathogenesis of FM is not completely known although a combination of genetic and environmental factors (i.e., psychological and physical stressors), neurotransmitters, hormones, cytokines, and immune factors seem to be involved [6]. 
The principal mechanism apparently implicated in the pathogenesis of the disease is the central sensitization characterized by enhanced peripheral nociceptive input as well as abnormal central pain processing of the central nervous system (CNS) [7]. This mechanism is responsible of hyperalgesia (increased sensitivity to pain) and allodynia (pain due to a non-painful stimulus), peculiar of FM.

Anotherpathogenetic hypothesis involves the hypothalamicpituitary-adrenal (HPA) axis dysfunction, which is responsible for an altered stress response [8]. Moreover, together with autonomic nervous system (ANS), the HPA axis contributes to preserve homeostasis through the release of neurotransmitters [9]. Many authors suggested the presence of a linkage among the HPA axis, ANS, T-lymphocytes, and cytokines and focused their research on profiling cytokines or on studying hormone dysfunctions in FM patients, but results were contradictory [10].

The prevalence of FM in first-degree relatives of FM patients has been also evaluated [11]. Other authors analyzed family aggregation of FM showing also a co-aggregation of FM with mood disorders in families; these data suggest that probably genetic factors are involved in the etiology of the disease [12]. Also, different polymorphisms could contribute to FM susceptibility, although findings were discordant [13].

Therefore, owing to the lack of specific biomarkers able to characterize different aspect of FM disease, the diagnosis of FM is made entirely on clinical features.

In the last few years, the discovery of extracellular microRNAs (miRNAs) in biological fluids (circulating miRNAs) has generated a great interest for their potential use as biomarkers [14]. MiRNAs are short ( 22 nt in length) non-coding RNAs that act as post-transcriptional regulators in many tissues, both in physiological and pathological conditions [15]. Bioinformatics tools predicted the presence of hundreds mRNA targets for each miRNA [16], suggesting that many genes are subject to miRNA-mediated regulation. Circulating miRNAs are stable and resistant to RNAses digestion, extreme $\mathrm{pH}$, high temperatures, extended storage, and multiple freeze-thaw cycles, and can be reliably analyzed by quantitative PCR (qPCR) assays [17].

Recently, two profiling studies of circulating miRNAs, in cerebrospinal fluid [18] and in serum [19], have been reported with the aim to evaluate their potential as circulating biomarkers in FM, but no correlations with clinical parameters have been done. Similarly, the expression of miRNAs from peripheral blood mononuclear cells (PBMCs) indicated a marked downregulation of many miRNAs but no significant correlations with cardinal symptoms of FM could be identified. Moreover, in all these studies, little is known about the initial status (i.e., pharmacologic treatment with analgesics) of the patients before blood withdrawal. In fact, it is known that also drugs may influence the expression level of circulating miRNAs [20]. Therefore, in order to minimize the analgesicrelated effects on miRNA expression, we accurately selected
FM patients and analyzed the expression of serum and saliva miRNAs. The correlation of circulating miRNAs with clinimetric parameters allowed us to obtain further insights into FM disease.

\section{Methods}

\section{Patients}

During a 1-year period, 14 consecutive adult patients affected by FM, according to both the 1990 and 2010 ACR criteria [4, 5], referring for the first time to the Clinic for the Diagnosis and Therapy of FM of the Unit of Rheumatology, Sapienza University of Rome, complaining of pain VAS $>6$ (range from 0 to 10), with positivity of at least 14 TPs and free from drugs at the time of evaluation, were enrolled.

The use of drugs usually prescribed for FM, like tricyclic antidepressants, antiepileptic drugs, selective serotonin reuptake inhibitors (SSRI), serotonin-norepinephrine reuptake inhibitors (SNRI) was prohibited at the time of enrolment and if these drugs were taken before, 3 months of washout were required. Acetaminophen was permitted $48 \mathrm{~h}$ before blood sampling. In the same period, healthy donors (i.e., controls) were enrolled. All patients underwent clinical examination with TP count. Patients and controls completed the following questionnaires: Fibromyalgia Impact Questionnaire (FIQ), Fibromyalgia Assessment Status (FAS), Health Assessment Questionnaire (HAQ), and Zung Self-Rating Anxiety and Zung Self-Rating Depression Scale (ZSAS, ZSDS) in validated Italian version.

The FIQ is a self-administered instrument that evaluates the health status of women with FM. It is composed of 10 items that measure physical functioning, work status, depression, anxiety, sleep, pain, stiffness, fatigue, and well-being [21]. The FAS is a short self-administered questionnaire, composed of three items: pain, fatigue, and sleep disturbances. It provides a single composite measure of disease activity in FM patients [22]. The HAQ is one of the first self-report functional status measures and it is especially used in patients with arthritis; it is based on five patient dimensions: disability, pain, medication effects, costs of care, and mortality [23]. The questionnaires on anxiety and depression that we administered have been developed and validated by Zung $[24,25]$. The test of anxiety has the function of providing a quantitative measure of the level of anxiety currently present in an individual, whereas the test of depression serves to detect a depressive tendency in a subject.

Patients with inflammatory or autoimmune diseases, osteoarthritis, neurologic, or psychiatric disturbances were excluded from the study. The study was conducted according to the protocol and good clinical practice principles and the Declaration of Helsinki statements. All patients gave their informed consent and the study was approved by the local Ethical Committee. 
Sample Collection and RNA Isolation At the time of blood sampling, patients and controls were fasted. Blood samples from FM patients $(n=14)$ and healthy donors $(n=14)$ were collected in SST II Advance tubes (BD Biosciences) and kept at room temperature for $30 \mathrm{~min}$ to clot. Serum was obtained (1800 g for $15 \mathrm{~min}$ at $4{ }^{\circ} \mathrm{C}$ ), checked for hemolysis by spectrophotometry at $414 \mathrm{~nm}$ (Nanodrop 2000, Thermo Scientific) and stored in cryovials at $-80{ }^{\circ} \mathrm{C}$ until analysis. Blood samples were processed within $2 \mathrm{~h}$ after collection. Circulating miRNAs were extracted from serum $(200 \mu \mathrm{l})$ using miRCURY RNA Isolation Kit-Biofluids (Exiqon) following the manufacturer's instruction. Spike-ins (RNA spike-in kit, Exiqon) and carrier RNA (MS2, Roche) were added before sample extraction. RNA samples were stored at $-80^{\circ} \mathrm{C}$ until use.

Circulating miRNAs Patients and controls were divided into two age-matched groups ( $n=7$ each). For each group, sera (30 $\mu \mathrm{l}$ each) were pooled in a single sample $(210 \mu \mathrm{l})$. RNAs were extracted and reverse transcribed in 20- $\mu 1$ reactions using the miRCURY LNA ${ }^{\mathrm{TM}}$ Universal RT cDNA Synthesis Kit (Exiqon) and assessed using the Serum/Plasma Focus miRNA PCR Panel I+II (96-wells, Exiqon) by qPCR (7900HT, Applied Biosystems). The expression levels of selected miRNAs were further evaluated by single assays by using specific $\mathrm{LNA}^{\mathrm{TM}}$ PCR primer set. After evaluating the expression of miR-16-5p, miR-103a-3p, and miR-191-5p, only $\mathrm{miR}-16-5 \mathrm{p}$ was used for normalization.

\section{Statistical Analysis}

The raw $\mathrm{Cq}$ values from amplification curves were normalized by global mean using the GenEx qPCR analysis software (Exiqon ver 5). Statistically significant $(p<0.05)$ miRNAs with a fold change (FC) smaller than $-1.5(\mathrm{FC}<-1.5)$ and greater than 1.5 (FC > 1.5) compared to controls were obtained. Expression values of statistically significant miRNAs were correlated (Spearman's) with clinical and clinimetric parameters. Statistics, correlation analyses (SPSS ver.12) and receiver operating characteristics (ROC) curves for each miRNA (SigmaPlot Ver.10.0) were performed. To obtain a predictive model, we calculated a generalized linear model based on expression values of the statistically significant miRNAs (function $\mathrm{glm}$ in R Bioconductor) (http://www.bioconductor. org/) [26].

Bioinformatics Target Prediction of Dysregulated miRNAs and Gene Ontology

Target prediction was carried out by using an already reported procedure [27] that integrates the prediction of three different prediction algorithms (i.e., TargetScan, MiRanda, and PITA). The list of target genes was employed in further analyses. We also performed a bioinformatics analysis by miR-Ontology Database miRò (http://ferrolab.dmi.unict.it/miro/) to find the potential biological role of miRNA targets significantly dysregulated in patients compared to controls. We performed Gene Ontology, KEGG pathway enrichment analysis, and annotation by DAVID bioinformatics tool [28] to determine the biological processes and signaling pathways in which the predicted targets of the deregulated miRs were involved.

\section{Results}

Patients' Characteristics The study population consisted of 14 female patients, without diagnosis of autoimmune diseases, psychiatric disorders, and neuromuscular diseases, and 14 healthy controls matched for sex and age. The mean age was 43.8 years (range from 24 to 56 years) in FM group and 42.3 years (range from 28 to 56 years) in the control group. The mean age of onset of the disease was 40 years (range from 24 to 50 years). Family history was negative for FM. All patients underwent clinical examination with TP count, blood withdrawal, and they completed the questionnaires. No one in the groups was taking drugs. All enrolled patients received diagnosis of FM for the first time; thus, they had never received any specific drug treatment for FM. The clinical and clinimetric data of the two groups have been reported in Table 1. According to the ACR 2010 criteria, all patients had a widespread index (WPI) $>7$ and a symptom severity scale (SS) $>5$ (or WPI from 3 to 6 and SS $>9$ ) with a statistical

Table 1 Clinimetric data. Demographics and clinimetric characteristics of FM patients and healthy controls. Values were reported as mean values (minimum and maximum values are reported in parentheses)

\begin{tabular}{llll}
\hline & $\begin{array}{l}\text { Healthy controls } \\
(\mathrm{n}=14)\end{array}$ & $\begin{array}{l}\text { FM patients } \\
(\mathrm{n}=14)\end{array}$ & $\begin{array}{l}\text { T test } \\
\text { ( } \mathrm{p} \text { value })\end{array}$ \\
\hline Age (years $)$ & $42.3(28$ to 56$)$ & $43.8(24$ to 56$)$ & 0.652 \\
WPI & $1.9(0$ to 5$)$ & $11.6(6$ to 15$)$ & $<0.001$ \\
SS SCORE & $2.3(0$ to 5$)$ & $10.4(8$ to 12$)$ & $<0.001$ \\
TP & - & $15.5(12$ to 18$)$ & - \\
FIQ & $5.708(0$ to 23.43$)$ & $68.6(41.9$ to 99.0$)$ & $<0.001$ \\
VAS work ability & $0.2(0$ to 1$)$ & $7.5(4$ to 10$)$ & $<0.001$ \\
VAS pain & $0.4(0$ to 3$)$ & $8.4(5$ to 10$)$ & $<0.001$ \\
VAS tiredness & $0.8(0$ to 4$)$ & $8.6(5$ to 10$)$ & $<0.001$ \\
VAS sleep quality & $0.6(0$ to 2$)$ & $8.4(5$ to 10$)$ & $<0.001$ \\
VAS stiffness & $0.6(0$ to 4$)$ & $7.9(3$ to 10$)$ & $<0.001$ \\
VAS anxiety & $0.7(0$ to 3$)$ & $7.2(1$ to 10$)$ & $<0.001$ \\
VAS depression & $0.2(0$ to 1$)$ & $5.1(0$ to 10$)$ & $<0.001$ \\
HAQ & $0.2(0$ to 1$)$ & $1.0(0$ to 2.4$)$ & $<0.001$ \\
FAS & $1.4(0$ to 5$)$ & $8.0(5.3$ to 10$)$ & $<0.001$ \\
ZSAS & $27.8(20$ to 35$)$ & $50.1(36$ to 63$)$ & $<0.001$ \\
ZSDS & $28.0(20$ to 50$)$ & $48.1(35$ to 59$)$ & $<0.001$ \\
\hline
\end{tabular}


significant difference with the controls $(p<0.001)$. In order to set a homogeneous cohort of patients, we decided to include only subjects who complained about pain with VAS higher than six and that presented at least 14 TPs at physical examination. The FIQ total score was greater in the group of patients compared to the healthy controls $(p<0.001)$ as well as single visual analog scale (VAS) that compose it: VAS work ability, VAS pain, VAS tiredness, VAS sleep quality, VAS stiffness, VAS anxiety, VAS depression $(p<0.001)$. Moreover, all the other scores used (HAQ, FAS, ZSAS, ZSDS) were significantly higher in FM patients $(p<0.001)$.

Five miRNAs Are Differentially Expressed in the Pooled FM Sera The exploratory profiling of circulating miRNAs revealed that five miRNAs were significantly $(p<0.05)$ and differentially regulated in patients with FM compared to healthy controls (Table 2). In particular, three miRNAs (miR-10a-5p, miR-320b, and miR-424-5p) were upregulated (FC >1.59), whereas two miRNAs (miR-20a-3p and miR$139-5 \mathrm{p})$ were downregulated (FC <-1.57). Moreover, we have found that the expression levels of mir-10a-5p and mir$139 a-5 p$ were very low compared to the other miRNAs and quite close to the experimental limit of detection.

Six miRNAs Are Differentially Expressed in Individual FM Ser a To confirm the differential expression of those miRNAs found deregulated by the preliminary exploratory profiling, we decided to include other miRNAs in the analysis of individual samples. We selected some miRNAs that resulted up- or downregulated in one or another of the two pools (data not shown), or that belonged to the same miRNA family or cluster of those miRNAs found significantly deregulated in the profiling. In particular, we decided to include the following miRNAs: mir-1, mir-133a, mir-10b-5p, mir-143-3p, mir29b-2-5p, mir-346, and mir-320a. Therefore, we evaluated the expression of a total of 17 miRNAs, of which five were the controls (i.e., miR-23a-3p and miR-451a to evaluate hemolysis and miR-103, miR-191 and miR-16 as endogenous controls). We evaluated the stability of the three endogenous

Table 2 Differentially expressed miRNAs detected in the pooled sera from patients with FM after profiling

\begin{tabular}{lll}
\hline & \multicolumn{2}{l}{ FM patients } \\
\cline { 2 - 3 } miRNA name & $\begin{array}{l}\text { Relative quantity } \\
\text { (FC } \pm \text { St. Dev) }\end{array}$ & Significance ( $\mathrm{p}$ value) \\
\hline hsa-miR-10a-5p & $1.59 \pm 0.05$ & 0.026 \\
hsa-miR-320b & $1.65 \pm 0.05$ & 0.030 \\
hsa-miR-424-5p & $1.75 \pm 0.05$ & 0.036 \\
hsa-miR-20a-3p & $-2.92 \pm 0.05$ & 0.037 \\
hsa-miR-139-5p & $-1.57 \pm 0.05$ & 0.031 \\
\hline
\end{tabular}

controls by inspecting the relative variation (i.e., standard deviation) of $\mathrm{Cq}$ values among different samples, and we found (data not shown) that miR-16 was the most stable endogenous controls compared to the other two. Therefore, we employed this miRNA for further normalization and analysis. Of these miRNAs, only six miRNAs resulted significantly deregulated (Table 3). Interestingly, the expression level of analyzed miRNAs resulted downregulated when the normalization with miR-16 was applied. In particular, miR-1 showed the greatest downregulation $(\mathrm{FC}=-3.29)$. Of note, miR-23a-3p showed a significant downregulation $(\mathrm{FC}=-2.94)$ in $\mathrm{FM}$ patients.

Correlation Between miRNA Expression and Clinical Parameters in FM Patients We examined the correlation between the level of differentially expressed miRNAs in patients with FM and the clinical and clinimetric parameters used for the identification of the disease. Among all considered parameters, we found only a significant negative correlation $(r=-0.608$ and $p=0.036)$ between miR-320b and the ZSDS value.

Receiver Operating Characteristics (ROC) Curves To evaluate whether the significantly deregulated miRNAs could be used as predictors of FM disease, we calculated the receiver operating characteristic (ROC) curves for each miRNAs (Table 4 and Supplementary Information). We noticed that single miRNAs displayed an area under the curve (AUC) greater than $\sim 0.7$. miR-1 displayed the highest AUC (0.7970) with a specificity of $76.9 \%$ and a sensitivity of $72.7 \%$, whereas miR-133a displayed the lowest AUC (0.6859), a sensitivity of $53.8 \%$ but a specificity of $100 \%$ (Table 4 and Fig. 1). We therefore calculated a generalized linear model based on the statistically significant miRNAs in FM respect to the controls (Fig. 1). The best model was obtained by taking into account only five miRNAs: miR-23a-3p, miR-1, miR-133a, miR-346, and miR-320b. In our

Table 3 Differentially expressed miRNAs in individual FM sera

\begin{tabular}{lll}
\hline & FM patients & \\
\cline { 2 - 3 } & $\begin{array}{l}\text { Relative } \\
\text { Quantity (FC } \pm \text { St. Dev) }\end{array}$ & $\begin{array}{l}\text { Significance } \\
\text { (p value) }\end{array}$ \\
\hline hsa-miR-23a-3p & $\begin{array}{ll}0.34 \pm 0.13 \\
(-2.94)\end{array}$ & 0.015 \\
hsa-miR-1 & $\begin{array}{l}0.30 \pm 0.10 \\
(-3.29)\end{array}$ & 0.013 \\
hsa-miR-133a & $\begin{array}{l}0.40 \pm 0.10 \\
(-2.49)\end{array}$ & 0.037 \\
hsa-miR-346 & $\begin{array}{l}0.56 \pm 0.04 \\
(-1.80)\end{array}$ & 0.032 \\
hsa-miR-139-5p & $\begin{array}{l}0.48 \pm 0.05 \\
(-2.09)\end{array}$ & 0.029 \\
hsa-miR-320b & $\begin{array}{l}0.63 \pm 0.02 \\
(-1.59)\end{array}$ & 0.008 \\
\hline
\end{tabular}


Table 4 Receiver operating characteristics (ROC). Area under the curve (AUC) for each miRNA is reported together with the correspondent sensitivity and specificity

\begin{tabular}{lccc}
\hline & AUC & Sensitivity (\%) & Specificity (\%) \\
\hline hsa-miR-23a-3p & 0.769 & 61.5 & 92.3 \\
hsa-miR-1 & 0.797 & 72.7 & 76.9 \\
hsa-miR-133a & 0.686 & 53.8 & 100.0 \\
hsa-miR-346 & 0.724 & 50.0 & 92.3 \\
hsa-miR-139-5p & 0.736 & 41.7 & 100.0 \\
hsa-miR-320b & 0.782 & 75.0 & 84.6 \\
Model (5 miRNAs) & 0.954 & 100.0 & 83.3 \\
\hline
\end{tabular}

experimental set-up, this model was able to achieve a $100 \%$ sensitivity and a high specificity $(83.3 \%)$ (Fig. 1). The addition of miR-139-5p did not increase or modify the predictive capability of the model and it was therefore not considered in the analysis.

Putative Targets of Deregulated miRNAs and Their Function We carried out a bioinformatics analysis to identify the gene targets of deregulated miRNAs, by following an already reported procedure that exploits the predictions of three different algorithms (TargetScan, MiRanda, and Pita) [27]. The genes, putatively targeted by dysregulated miRNAs in FM, belong to different pathways and biological processes related to the disease (Fig. 2). Among the significant pathways (Benjamini and Hochberg post hoc corrected pvalues), we found tight junction ( $p$ value $=0.021$ ) and adherens junction ( $p$ value $=0.015)$, whereas the biological processes were related to transcription $(p$ value $=1.32 \mathrm{E}-04)$ and regulation of transcription ( $p$ value $=3.91 \mathrm{E}-05$ ), regulation of RNA metabolic process $(p$ value $=0.001)$, and

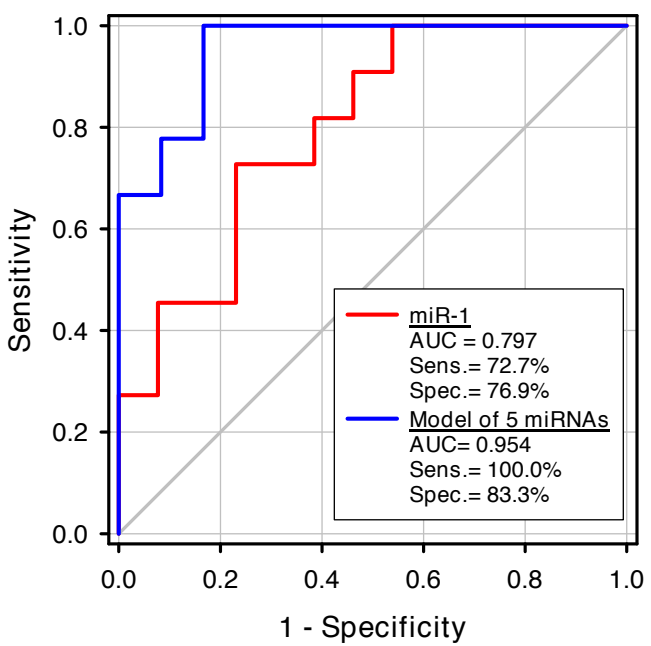

Fig. 1 Receiver operating characteristic (ROC) curve of miR-1 (red) and of the cumulative model (blue) obtained by the combination of five different miRNAs (i.e., miR-23a-3p, miR-1, miR-133b, miR-346, and miR320b) intracellular receptor-mediated signaling pathway ( $p$ val$\mathrm{ue}=0.030$ ). Interestingly, many target genes were related to the neurotrophin signaling pathway (Table S1) and axon guidance (Table S2), neurotransmitter transporter activity (Table S3) or were found as synapse (Table S4) or cell cortex components (Table S5).

Expression Levels of Circulating miRNAs in Saliva of FM Patients Saliva has been considered another potential source for liquid biopsies. To evaluate if the saliva of patients affected by FM also contain the same dysregulated miRNAs found in their sera, we analyzed the expression levels only of the six statistically dysregulated miRNAs. Interestingly, only a half of miRNAs (miR-23a-3p, miR-346, and miR-320b) were expressed in saliva. However, their expression values were not statistically different compared to controls.

\section{Discussion}

The discovery of the upregulation of miR-155 and miR-146a in animal models of rheumatoid arthritis prompted the study of the role of miRNAs in other inflammatory pain syndromes or as a potential therapeutic strategy [29]. In the last few years, the fundamental role of miRNAs in the regulation of pain disorders have been reported [30]. The alteration of protein expression, potentially regulated by microRNAs, has been hypothesized to be one of the principal culprits of the central and peripheral sensitization in patients affected by painful disorders (i.e., complex regional pain syndrome, irritable bowel disorder, endometriosis, osteoarthritis of knee and FM) [30]. This process is necessary to develop and maintain chronic pain as in FM syndrome [31]. Other studies focused the attention on the involvement of miRNAs in chronic pain because of the regulation of specific pain-related genes [32, 33]. However, the pathophysiological mechanism in FM is ambiguous, and the Bneurogenic landscape ${ }^{\wedge}$ and the activation of nociceptive pathways are unclear.

Despite extensive efforts in the FM field, clinicians do not have unambiguous diagnostic techniques available so far. Therefore, in recent years, few studies were aimed at exploring biomarkers of FM by examining the expression profiles of miRNAs circulating in biofluids such as cerebrospinal fluid (CSF) [18] and serum [19]. However, the results of the latter two studies emphasized the correlation of some FM biomarkers with pain and fatigue. In the first study, the authors identified nine downregulated miRNAs (miR-21-5p, miR-145-5p, miR-29a-3p, miR-99b-5p, miR125b-5p, miR-23a-3p, miR-23b-3p, miR-195-5p, miR223-3p) in CSF respect to control subjects but only miR145-5p, which is strongly deregulated in FM, was associated with FIQ pain and FIQ fatigue, two characteristic symptoms of FM. A limitation of that work was the lack 
Fig. 2 Biological processes of the genes targeted by the dysregulated miRNAs found in FM patients compared to controls

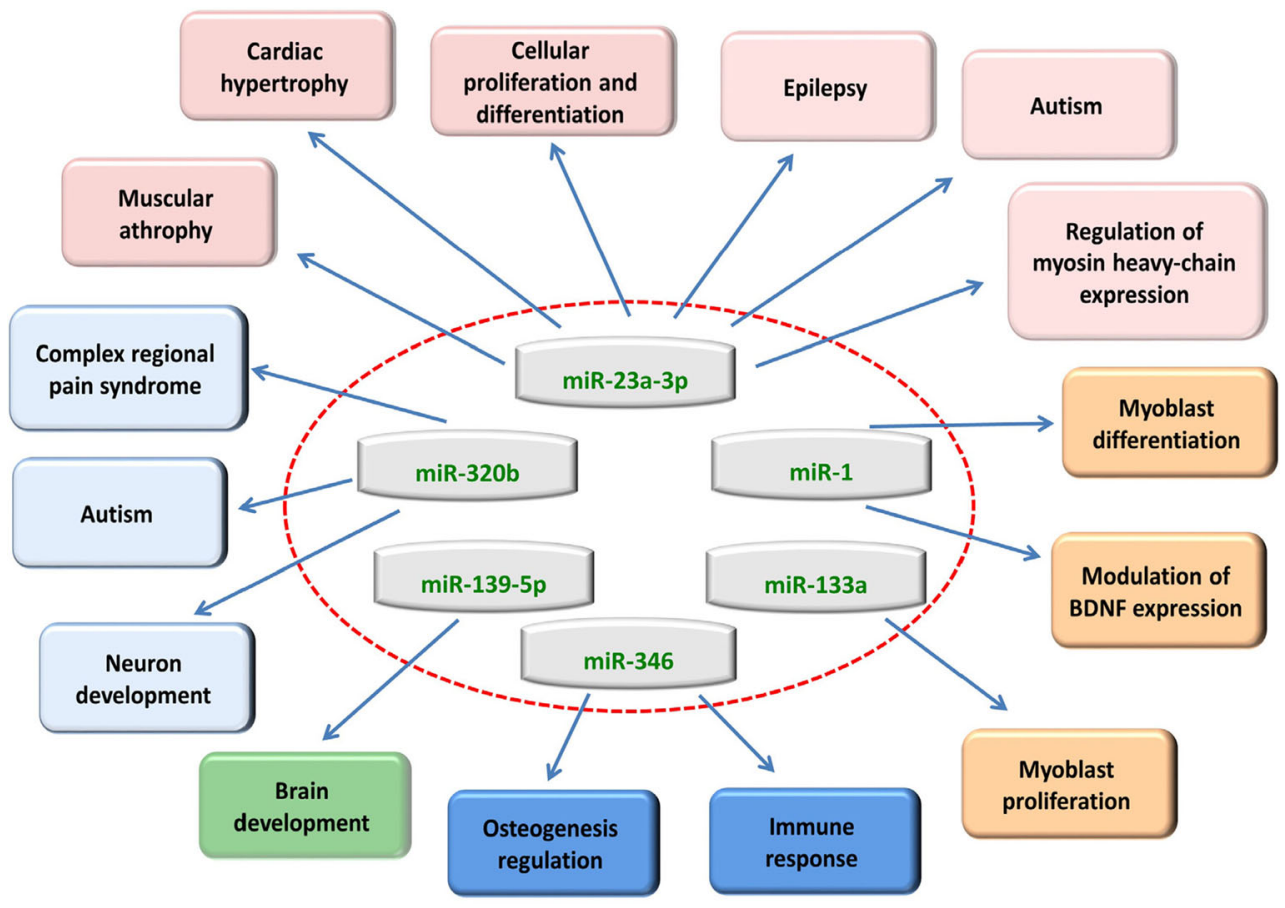

of bioinformatics analyses aimed at predicting the putative target genes of dysregulated miRNAs, thus limiting the impact only on the pathophysiological interpretation of FM. In the second study, the authors reported that eight miRNAs were differentially expressed in FM patients, seven were downregulated (miR-103a-3p, miR-107, let-7a-5p, miR-30b-5p, miR-151a-5p, miR-142-3p, and miR-374b$5 p$ ) and only one, namely miR-320a, was upregulated compared to healthy controls [19]. However, the major limitation of that work was the use of a pharmacologic treatment with monoamine reuptake inhibitors (i.e., SSRI and SNRI), which represents a severe confounding factor to miRNAs analysis and endogenous expression values. In fact, drugs may affect severely the expression level of circulating miRNAs [20, 34]. Therefore, an accurate selection of patients before blood withdrawal is mandatory.

Another recent study examined the expression profiles of miRNAs to build up a quantitative diagnostic method of FM, but the authors performed the study on peripheral blood mononuclear cells (PBMCs) and not on circulating miRNAs [35]. In any case, although not perfectly comparable with our or the other two studies on circulating miRNAs, the authors identified a marked downregulation (at least twofold) of five miRNAs (miR-223-3p, miR-451a, miR-338-3p, miR-143-3p, and miR-145-5p).

Interestingly, whatever the biofluids or specimens considered (i.e., serum, CSF, or PBMCs), the expression levels of deregulated miRNAs in FM is always lower than that of the controls, suggesting a potential implication in FM biological processes.
A common problem of all of these investigations is the small number of FM patients analyzed. In our case, to find a selected Bnovel-diagnosed ${ }^{\wedge}$ population without previous pharmacological treatments was not trivial. Obviously, we will need to extend the exploration to a wider cohort of patients to validate the results that we have found in this work. Moreover, the dysregulation of miRNAs in circulation could be either a cause or a consequence of the disease, preventing a clear attribution of the role of these RNA species. Also the bioinformatics prediction of miRNA targets is meaningful only by assuming that these circulating miRNAs, to exploit their role, should be internalized within the recipient cells where they can bind to mRNAs.

Despite all of these limitations, we emphasize that also in our exploratory study, all the significantly modulated miRNAs are downregulated. In particular, we found six downregulated miRNAs (miR-23a-3p, miR-1, miR-133a, miR-346, miR-139-5p, and miR-320b) in FM patients compared to controls. Interestingly, miR-320b showed a significant negative correlation with ZSDS value that represents a score for depressive symptoms reported by patients. This association, never reported before, is suggestive of a functional involvement of this miRNA, as depression is a very common symptom in FM. Previously, this miRNA has been found inversely correlated only with pain [19]. Moreover, miR-320 has been reported to promote neurite outgrowth, play a key role in neuronal development, and improve neuronal regeneration after injury [36]. Mir-320 is also deregulated in whole blood of patients with complex regional pain syndrome, a disease caused by a dysfunction in the central and/or 
peripheral nervous systems and characterized by chronic neuropathic pain [37].

Interestingly, we found a downregulation of miR-23a which is, instead, generally considered a stable miRNA normalizer for relative quantification in tissues [38] and in plasma/serum [39]. MiR-23a was found downregulated also in CSF of FM patients, although not correlated with the cardinal symptoms of FM [18]. MiR-23a is the first member of the cluster miR-23a/ 27a/24-2 and was reported to downregulate MURF1 (an antihypertrophic protein leading to cardiac hypertrophy) and MAFbx (an atrophic factor) that provides resistance to muscle atrophy [40]. Therefore, this miRNA could have a role in the maintenance of skeletal muscle integrity, because it targets key molecules regulating contractile and metabolic properties of skeletal muscle, myosin heavy-chains, and peroxisome proliferator-activated receptor gamma [41].

Interestingly, we found a dysregulation of miR-1 and miR133a in FM patients. Recently, the role of miR-1 in modulating the expression of the Bbrain-derived neurotrophic factor ${ }^{\wedge}$ (BDNF) has been highlighted [42]. BDNF is a protein belonging to the family of neurotrophins that has important functions not only in brain development and synaptogenesis [43], but also in learning and memory [44], and myogenesis [45]. The alteration of BDNF has been associated with many nervous system disorders including Huntington's disease, depression, and anxiety [42]. In fact, an adequate level of BDNF are needed to adjust the number of dopaminergic neurons in the mesencephalic substantia nigra, whereas the regulation of BDNF expression in the hippocampus is a crucial process in memory and learning [42].

The upregulation of circulating miR-139-5p, miR-1, and miR-133a in healthy, trained men during endurance exercises and training activities has been previously reported [46, 47]. The reduced physical activity of FM patients because of pain and fatigue symptoms may explain, at least in part, the downregulation of these miRNAs in our patients.

Circulating miR-139-5p has been also analyzed in stressrelated psychiatric disorders, where the expression is reduced in rats resilient to stress conditions [48].

In recent years, the first study carried out on schizophrenia patients emphasized the slightly reduced expression of miR-346, suggesting that along with other miRNAs, miR-346 may have a strong diagnostic potential for neurological diseases [49].

Finally, all of the miRNAs dysregulated in our patients have several links with the biology and functioning of the central nervous system, skeletal muscle or have many connections with physical activity, pain, stress, mood disorders, and depressive symptoms. Moreover, we suggest that the combination of several dysregulated miRNAs may represent the basis to obtain a good predictive model with high diagnostic power. The choice of the Bliquid biopsy^ (i.e., CSF, serum, saliva) is another variable to consider to reduce variability. In fact, we did not find any correlation between serum and saliva circulating miRNAs, whereas others did not find any overlap between serum and CSF $[18,19]$.
In conclusion, our study, although limited to a small cohort, emphasizes that the significant dysregulation of several serum-circulating miRNAs linked to FM disease may have neurological and muscular origin. Moreover, further studies should be dedicated to strengthen these preliminary findings and to explore whether our results can apply also to juvenile fibromyalgia [1-3].

Finally, considering that in this first study, we recruited only patients Bnaïve ${ }^{\wedge}$ to therapy, it would be interesting to evaluate the behavior of miRNAs after treatment.

Acknowledgments The authors thank Dr. Letizia Da Sacco for technical discussions about the quantification of circulating miRNAs from serum.

Compliance with Ethical Standards The study was conducted according to the protocol and good clinical practice principles and the Declaration of Helsinki statements. All patients gave their informed consent and the study was approved by the local Ethical Committee.

Funding This work was supported by the SAPIENZA University of Rome to M.D.F, by the Italian Ministry of Health to A.M. and by the Italian Ministry for Education, University and Research in the framework of the Flagship Project NanoMAX to C.B.

Conflict of Interest The authors declare that they have no competing interests.

\section{References}

1. Kashikar-Zuck S, King C, Ting TV and Arnold LM (2016) Juvenile Fibromyalgia: Different from the Adult Chronic Pain Syndrome?. Curr. Rheumatol. Rep. 18, 19-016-0569-9

2. Buskila D, Press J, Gedalia A, Klein M, Neumann L, Boehm R, Sukenik S (1993) Assessment of nonarticular tenderness and prevalence of fibromyalgia in children. J Rheumatol 20:368-370

3. Gerloni V, Ghirardini M, Fantini F (1998) Assessment of nonarticular tenderness and prevalence of primary fibromyalgia syndrome in healthy Italian schoolchildren. Arthritis Rheum 41:1405

4. Wolfe F, Smythe HA, Yunus MB, Bennett RM, Bombardier C, Goldenberg DL, Tugwell P, Campbell SM et al (1990) The American College of Rheumatology 1990 criteria for the classification of fibromyalgia. Report of the Multicenter Criteria Committee Arthritis Rheum 33:160-172

5. Wolfe F, Clauw DJ, Fitzcharles MA, Goldenberg DL, Katz RS, Mease P, Russell AS, Russell IJ et al (2010) The American College of Rheumatology preliminary diagnostic criteria for fibromyalgia and measurement of symptom severity. Arthritis Care Res (Hoboken) 62:600-610

6. Sarzi-Puttini P, Atzeni F, Mease PJ (2011) Chronic widespread pain: from peripheral to central evolution. Best Pract Res Clin Rheumatol 25:133-139

7. Yunus MB (2008) Central sensitivity syndromes: a new paradigm and group nosology for fibromyalgia and overlapping conditions, and the related issue of disease versus illness. Semin Arthritis Rheum 37:339-352

8. Crofford LJ (2002) The hypothalamic-pituitary-adrenal axis in the pathogenesis of rheumatic diseases. Endocrinol Metab Clin N Am 31:1-13

9. Di Franco M, Iannuccelli C, Valesini G (2010) Neuroendocrine immunology of fibromyalgia. Ann N Y Acad Sci 1193:84-90 
10. Rodriguez-Pinto I, Agmon-Levin N, Howard A, Shoenfeld Y (2014) Fibromyalgia and cytokines. Immunol Lett 161:200-203

11. Buskila D, Neumann L (1997) Fibromyalgia syndrome (FM) and nonarticular tenderness in relatives of patients with FM. J Rheumatol 24:941-944

12. Arnold LM, Hudson JI, Hess EV, Ware AE, Fritz DA, Auchenbach MB, Starck LO, Keck PE Jr (2004) Family study of fibromyalgia. Arthritis Rheum 50:944-952

13. Lee YH, Choi SJ, Ji JD, Song GG (2012) Candidate gene studies of fibromyalgia: a systematic review and meta-analysis. Rheumatol Int 32:417-426

14. Cortez MA, Calin GA (2009) MicroRNA identification in plasma and serum: a new tool to diagnose and monitor diseases. Expert Opin Biol Ther 9:703-711

15. Bartel DP (2009) MicroRNAs: target recognition and regulatory functions. Cell 136:215-233

16. Tarang S, Weston MD (2014) Macros in microRNA target identification: a comparative analysis of in silico, in vitro, and in vivo approaches to microRNA target identification. RNA Biol 11:324-333

17. Chen Y., Gelfond J. A., McManus LM and Shireman PK (2009) Reproducibility of quantitative RT-PCR array in miRNA expression profiling and comparison with microarray analysis. BMC Genomics. 10, 407-2164-10-407

18. Bjersing JL,Lundborg C, Bokarewa MI, Mannerkorpi K (2013) Profile of cerebrospinal microRNAs in fibromyalgia. PLoS One 8:e78762

19. Bjersing JL, Bokarewa MI, Mannerkorpi K (2015) Profile of circulating microRNAs in fibromyalgia and their relation to symptom severity: an exploratory study. Rheumatol Int 35:635-642

20. de Boer HC, van Solingen C, Prins J, Duijs JM, Huisman MV, Rabelink TJ, van Zonneveld AJ (2013) Aspirin treatment hampers the use of plasma microRNA-126 as a biomarker for the progression of vascular disease. Eur Heart J 34:3451-3457

21. Burckhardt CS,Clark SR, BennettRM (1991) The fibromyalgia impact questionnaire: development and validation. J Rheumatol 18:728-733

22. Iannuccelli C, Sarzi-Puttini P, Atzeni F, Cazzola M, di Franco M, Guzzo MP, Bazzichi L, Cassisi GA et al (2011) Psychometric properties of the fibromyalgia assessment status (FAS) index: a national web-based study of fibromyalgia. Clin Exp Rheumatol 29:S49-S54

23. Bruce B, Fries JF (2005) The health assessment questionnaire (HAQ). Clin Exp Rheumatol 23:S14-S18

24. Zung WW (1971) A rating instrument for anxiety disorders. Psychosomatics 12:371-379

25. Zung WW (1972) The depression status inventory: an adjunct to the self-rating depression scale. J Clin Psychol 28:539-543

26. Gentleman RC, Carey VJ, Bates DM, Bolstad B, Dettling M, Dudoit S, Ellis B, Gautier L et al (2004) Bioconductor: open software development for computational biology and bioinformatics. Genome Biol 5:R80

27. Masotti A, Alisi A (2012) Integrated bioinformatics analysis of microRNA expression profiles for an in-depth understanding of pathogenic mechanisms in non-alcoholic fatty liver disease. $\mathrm{J}$ Gastroenterol Hepatol 27:187-188

28. da Huang W, Sherman BT, Lempicki RA (2009) Systematic and integrative analysis of large gene lists using DAVID bioinformatics resources. Nat Protoc 4:44-57

29. Ceribelli A, Nahid MA, Satoh M, Chan EK (2011) MicroRNAs in rheumatoid arthritis. FEBS Lett 585:3667-3674

30. Andersen HH, Duroux M, Gazerani P (2014) MicroRNAs as modulators and biomarkers of inflammatory and neuropathic pain conditions. Neurobiol Dis 71:159-168

31. Gold MS, Gebhart GF (2010) Nociceptor sensitization in pain pathogenesis. Nat Med 16:1248-1257

32. Bai G, Ambalavanar R, Wei D, Dessem D (2007) Downregulation of selective microRNAs in trigeminal ganglion neurons following inflammatory muscle pain. Mol Pain 3:15
33. Poh KW, Yeo JF, Ong WY (2011) MicroRNA changes in the mouse prefrontal cortex after inflammatory pain.Eur J Pain 15:801 e1-801.12

34. Russo F, Di Bella S, Bonnici V, Lagana A, Rainaldi G, Pellegrini M, Pulvirenti A, Giugno R and Ferro A (2014) A knowledge base for the discovery of function, diagnostic potential and drug effects on cellular and extracellular miRNAs. BMC Genomics. 15 Suppl 3, S4-2164-15-S3-S4

35. Cerda-Olmedo G, Mena-Duran AV, Monsalve V, Oltra E (2015) Identification of a microRNA signature for the diagnosis of fibromyalgia. PLoS One 10:e0121903

36. White RE, Giffard RG (2012) MicroRNA-320 induces neurite outgrowth by targeting ARPP-19. Neuroreport 23:590-595

37. Orlova IA, Alexander GM, Qureshi RA, Sacan A, Graziano A, Barrett JE, Schwartzman RJ and Ajit SK, (2011) MicroRNA modulation in complex regional pain syndrome. J. Transl. Med. 9, 195-58769-195

38. Shen Y, Li Y, Ye F, Wang F, Wan X, Lu W, Xie X (2011) Identification of miR-23a as a novel microRNA normalizer for relative quantification in human uterine cervical tissues. Exp Mol Med 43:358-366

39. Blondal T, Jensby NS, Baker A, Andreasen D, Mouritzen P, Wrang TM, Dahlsveen IK (2013) Assessing sample and miRNA profile quality in serum and plasma or other biofluids. Methods 59:S1-S6

40. Chhabra R, Dubey R and Saini N (2010) Cooperative and individualistic functions of the microRNAs in the miR-23a 27a 24-2 cluster and its implication in human diseases. Mol. Cancer. 9, 232-45989-232

41. Wada S, Kato Y, Sawada S, Aizawa K, Park JH, Russell AP, Ushida T, Akimoto T (2015) MicroRNA-23a has minimal effect on endurance exercise-induced adaptation of mouse skeletal muscle. Pflugers Arch 467:389-398

42. Varendi K, Kumar A, Harma MA, Andressoo JO (2014) miR-1, miR-10b, miR-155, and miR-191 are novel regulators of BDNF. Cell Mol. Life Sci 71:4443-4456

43. Huang EJ, Reichardt LF (2001) Neurotrophins: roles in neuronal development and function. Annu Rev Neurosci 24:677-736

44. Nakajo Y, Miyamoto S, Nakano Y, Xue JH, Hori T, Yanamoto H (2008) Genetic increase in brain-derived neurotrophic factor levels enhances learning and memory. Brain Res 1241:103-109

45. Mousavi K, Jasmin BJ (2006) BDNF is expressed in skeletal muscle satellite cells and inhibits myogenic differentiation. J Neurosci 26:5739-5749

46. Nielsen S, Scheele C, YfantiC, Akerstrom T,Nielsen AR, Pedersen BK, Laye MJ (2010) Muscle specific microRNAs are regulated by endurance exercise in human skeletal muscle. J Physiol 588:4029-4037

47. Nielsen S, Akerstrom T, Rinnov A, Yfanti C, Scheele C, Pedersen BK, Laye MJ (2014) The miRNA plasma signature in response to acute aerobic exercise and endurance training. PLoS One 9:e87308

48. Chen RJ, Kelly G, Sengupta A, Heydendael W, Nicholas B, Beltrami S, Luz S, Peixoto L et al (2015) MicroRNAs as biomarkers of resilience or vulnerability to stress. Neuroscience $305: 36-48$

49. Shi W, Du J, Qi Y, Liang G, Wang T, Li S, Xie S, Zeshan B et al (2012) Aberrant expression of serum miRNAs in schizophrenia. J Psychiatr Res 46:198-204

\section{Key Messages}

- Serum miRNAs correlate with major fibromyalgia (FM) symptoms

- A statistical linear model made of five miRNAs could discriminate FM patients from controls

- Liquid biopsies collected from different body parts have different miRNA profiles 